\title{
Effect of tanshinone IIA on oxidative stress and apoptosis in a rat model of fatty liver
}

\author{
GUAN-LIN YANG, LIAN-QUN JIA, JIN WU, YI-XIN MA, HUI-MIN CAO, NAN SONG and NI ZHANG \\ Key Laboratory of Ministry of Education for TCM Viscera-State Theory and Applications, \\ Liaoning University of Traditional Chinese Medicine, Shenyang, Liaoning 110847, P.R. China
}

Received January 4, 2016; Accepted February 14, 2017

DOI: $10.3892 /$ etm.2017.5162

\begin{abstract}
Oxidative stress is a crucial factor associated with fatty liver disease, which raises the possibility of using antioxidants to improve liver steatosis. Tanshinone IIA (TSIIA) is a traditional Chinese medicine that has been reported to have antioxidant effects in vitro. The present study aimed to investigate whether TSIIA possesses antioxidant effects in vivo and whether TSIIA was able to improve liver steatosis. Hence, the ability of TSIIA to protect rats from liver disease was explored, particularly in regard to antioxidant activity. Rats were fed a high-lipid diet for 90 days, causing severe liver steatosis, both morphologically and biochemically. An increase in reactive oxygen species (ROS) in the liver was exhibited in addition to significantly elevated serum lipids and malondialdehyde (MDA). Furthermore, hepatocyte apoptosis was measured by Hoechst staining, reverse transcription-quantitative polymerase chain reaction and western blot analysis and an increase in hepatocyte apoptosis rate was indicated in mice on a high-fat diet. Following intraperitoneal injection of TSIIA (10 $\mathrm{mg} / \mathrm{kg} /$ day), liver steatosis was significantly inhibited. In rats receiving TSIIA treatment, less ROS were indicated in the liver and significantly decreased levels of MDA $(\mathrm{P}<0.05)$ in serum were exhibited, whereas significantly increased activities of total superoxide dismutase (T-SOD) and glutathione peroxidase (GSH-PX) were observed $(\mathrm{P}<0.05$ and $\mathrm{P}<0.01$, respectively). In addition, the rate of hepatocyte apoptosis was significantly decreased in the TSIIA group $(\mathrm{P}<0.01)$. However, TSIIA elicited no effect on serum lipid profiles. These results suggest that TSIIA attenuates oxidative stress by decreasing ROS and MDA production and enhancing the activity of
\end{abstract}

Correspondence to: Professor Guan-Lin Yang, Key Laboratory of Ministry of Education for TCM Viscera-State Theory and Applications, Liaoning University of Traditional Chinese Medicine, 79 Chongshan Easten Road, Huanggu, Shenyang, Liaoning 110847, P.R. China

E-mail: yang_guanlin@163.com

Key words: fatty liver, tanshinone IIA, free radical species, glutathione peroxidase, total superoxide dismutase, apoptosis
T-SOD and GSH-PX, which may contribute to the inhibition of apoptosis and amelioration of liver steatosis.

\section{Introduction}

Oxidative stress results from an imbalance between in vivo oxidative and anti-oxidative effects (1). Oxidative stress is involved in the pathophysiological processes of various diseases, including arteriosclerosis, diabetes, ischemia/reperfusion injury, ethanol intoxication and liver steatosis (2). When the body encounters harmful stimuli, reactive oxygen species (ROS) are produced that may not be effectively removed by antioxidants (3). ROS may be produced by non-alcoholic fatty liver disease (NAFLD) oxidase, xanthine oxidase and the mitochondrial electron transport system (4). Increased levels of ROS have been indicated to enhance the expression of apoptotic genes (5). Furthermore, apoptosis has been indicated as a prominent pathology of liver diseases and is a well-acknowledged type of programmed cell death (6). For protection against oxidative stress-related injury, the body possesses several scavenger systems, including enzymatic and non-enzymatic antioxidants. Enzymatic antioxidants include superoxide dismutase (SOD), catalase (CAT) and the selenium dependent enzyme, glutathione peroxidase (GSH-PX) (7). As antioxidant enzymes may affect lipid peroxidation, an increase in the activities of these enzymes may delay liver steatosis. Furthermore, oxidative stress has been indicated to induce structural and functional cellular effects through damage to DNA, proteins or membrane lipids (8). With respect to hepatocytes, lipid metabolism function may be impaired.

Clinically, liver steatosis without excessive alcohol consumption is known as NAFLD, which is the most common liver condition in the world (9). Abnormal lipid metabolism, which is a high risk for atherosclerosis, is a condition that may result in various types of cardiovascular diseases (10). Previous studies have demonstrated that steatotic livers have increased susceptibility to oxidative stress and that increased ROS may trigger an apoptotic cascade $(2,11)$. Although the scavenging mechanisms of the human body that act against excess ROS-induced cellular injury are typically sufficient, these may not always be enough to combat external stresses. Therefore, there is a pressing requirement to discover natural antioxidants (12). The dried roots of Salvia miltiorrhiza Bge, a perennial herb in the mint family, are used as a traditional 
Chinese medicine. In China, the herb is used as an adjunct therapy to treat particular circulatory diseases by promoting blood circulation and removing blood stasis (13). Tanshinone IIA (TSIIA) is one of the major lipophilic components isolated from Salvia miltiorrhiza Bge (14). Previous clinical trials and experimental studies have reported the antioxidant and anti-apoptotic properties of TSIIA $(15,16)$. As a natural antioxidant, TSIIA is able to protect cardiac myocytes through anti-oxidative pathways, inhibit oxidation of LDL in vitro and reduce the serum levels of oxidized low-density lipoprotein in mice (17-19). In our previous study, we showed that TSIIA was able to ameliorate atherosclerosis by suppressing toll-like receptor 4 (TLR4) and tumor necrosis factor (TNF)- $\alpha$ expression, in vitro (20). Large-scale prospective studies have also shown that TSIIA can be beneficial for cardiovascular diseases (14-16). However, there are few studies on the specific mechanism of TSIIA activity on the liver. We have previous indicated that TSIIA is able to protect cells from apoptosis in vitro (21). Thus, the purpose of the present study was to evaluate the effects of TSIIA on oxidative stress and hyperlipidemia in rats with fatty livers and to further explore the mechanism of action of TSIIA.

\section{Materials and methods}

Pharmacological agents, chemicals and reagents. Sodium TIIA sulfonate was purchased from Shanghai No. 1 Biochemical Pharmaceutical Co., Ltd. (Shanghai, China). Lactate dehydrogenase (LDH), MDA, peroxidase (POD), T-SOD and creatine kinase (CK) test kits were obtained from Nanjing Jiancheng Bioengineering Institute (Nanjing, China). Total cholesterol (TC), thyroglobulin (TG), low density lipoprotein cholesterol (LDL-C), high density lipoprotein cholesterol (HDL-C) test kits were purchased from Sichuan Maker Biotechnology Co., Ltd. (Chengdu, China). TRIzol reagent and the reverse transcription-quantitative polymerase chain reaction (RT-qPCR) kit were purchased from Takara Biotechnology Co., Ltd. (Dalian, China). The BCA Protein Assay kit, SDS-PAGE Gel Preparation kit and all horseradish peroxidase-conjugated secondary antibodies were purchased from Santa Cruz Biotechnology, Inc. (Dallas, TX, USA). RIPA lysis buffer was purchased from Beyotime Institute of Biotechnology (Shanghai, China).

Animals. A total of 90 male Sprague-Dawley rats $(300 \pm 10 \mathrm{~g})$ aged 6 weeks were purchased from Vital River Laboratories (Beijing, China). Rats were maintained under standard conditions of 12 -h light-dark cycle (temperature, $22 \pm 1^{\circ} \mathrm{C}$; humidity, $50 \pm 5 \%$ ) with free access to water. All animal studies were approved by the Local Ethics Committee for Animal Experimentation (approval number 2012-113), according to the guidelines of the Animal Committee of Liaoning University of Traditional Chinese Medicine (Shenyang, China). All efforts were made to minimize the number of animals used and their suffering throughout the study. Rats were randomly divided into a control (CON) group, a high-fat diet group (HFD) group and a TII A treatment (TAN) group ( $\mathrm{n}=30$ per group). Rats in the CON group were fed a regular balanced diet, while those in the HFD group and TAN group were fed a high-fat diet (6.0\% sucrose, $1.0 \%$ sodium glutamate, $5.0 \%$ yolk powder,
$8.0 \%$ peanut oil, $1.5 \%$ cholesterol, $0.4 \%$ methylthiouracil, $0.2 \%$ sodium cholate and $77.9 \%$ regular balanced diet) for 3 months. Furthermore, rats in the TAN group received $1.2 \mathrm{ml}$ sodium TIIA sulfonate $(10 \mathrm{mg} / \mathrm{kg})$ by intraperitoneal injection once a day in the last 2 months, while those in the control group and the HFD group received the same quantity of normal saline for the same duration of time. At 3 months, the rats were sacrificed by cervical dislocation under $10 \%$ choral hydrate anesthesia (TCI Development Co, Ltd., Shanghai, China), and small portions of hepatic tissue were excised from the right lobe of the liver.

High-resolution ultrasound imaging. Rats were anaesthetized with isoflurane (Beijing Zhongsheng Ruitai Technology Co., Ltd., Beijing, China) (1.5-3.0\%), together with continuous oxygen $(1 \mathrm{l} / \mathrm{min})$. Lipid deposition in the liver was observed using a Vevo 2100 High-Resolution Imaging System (FUJIFILM VisualSonics Inc., Toronto, Canada) according to the manufacturer's instructions.

Serum LDH, MDA, POD, T-SOD, CK and GSH-PX content. Rats were anaesthetized with $10 \%$ chloral hydrate. Aortic blood was collected and the samples were left to stand for $2 \mathrm{~h}$ at room temperature prior to centrifugation at $4{ }^{\circ} \mathrm{C}$ at $1,000 \mathrm{xg}$ for $20 \mathrm{~min}$. Serum was collected and stored at $-80^{\circ} \mathrm{C}$. Serum LDH, MDA, POD, T-SOD, CK, and GSH-PX content were estimated using commercial assay kits (A020-1, A003-1, A084-3, A001-1, A032 and A005, all Nanjing Jiancheng Bioengineering Institute).

Lipid detection. A liver sample of $0.1 \mathrm{~g}$ was homogenized in $1 \mathrm{ml}$ normal saline, supplemented with $50 \mathrm{ml}$ of a 2:1 chloroform $(\geq 99.7 \%)$ to methanol ( $\geq 99.0 \%)$ mixture and left to stand for $10 \mathrm{~min}$. Subsequently, the sample was centrifuged at $4^{\circ} \mathrm{C}$ at $3,000 \mathrm{xg}$ for $10 \mathrm{~min}$ and the upper layer was removed. The remaining sample was dried using a Termovap sample concentrator, resuspended in $0.5 \mathrm{ml}$ methanol $(\geq 99.0 \%)$ and examined using an automatic biochemical analyzer (Toshiba Medical Systems Corp., Otawara-Shi, Japan). TC and TG levels were measured by the COD-CE-PAP and GPO-PAP methods (22), respectively. Serum TG, TC, HDL-C, LDL-C levels were determined by corresponding assay kits $(0615021,0815031$, 0915051 and 0915061, all Sichuan Maker Biotechnology Co., Ltd., Sichuan, China).

Hematoxylin and eosin staining of liver tissues. Liver samples were fixed in $4 \%$ paraformaldehyde solution at room temperature for $24 \mathrm{~h}$ and stained with hematoxylin and eosin using standard techniques. The samples were embedded in paraffin, and $5-\mu \mathrm{m}$ thick paraffin sections were cut. The paraffin was removed with xylene, rehydrated through an alcohol gradient, and stained with hematoxylin and eosin and observed using light microscopy at magnification, x200. Images were captured using light microscope linked to a digital CCD camera (Olympus Corp., Tokyo, Japan).

Oil Red O staining of liver tissue. Oil Red O staining was performed following a standard protocol. Frozen liver sections (6- $\mu \mathrm{m}$ thick) were cut and dried. Samples were washed with $50 \%$ ethanol, stained with Oil Red O for 8 min, differentiated 

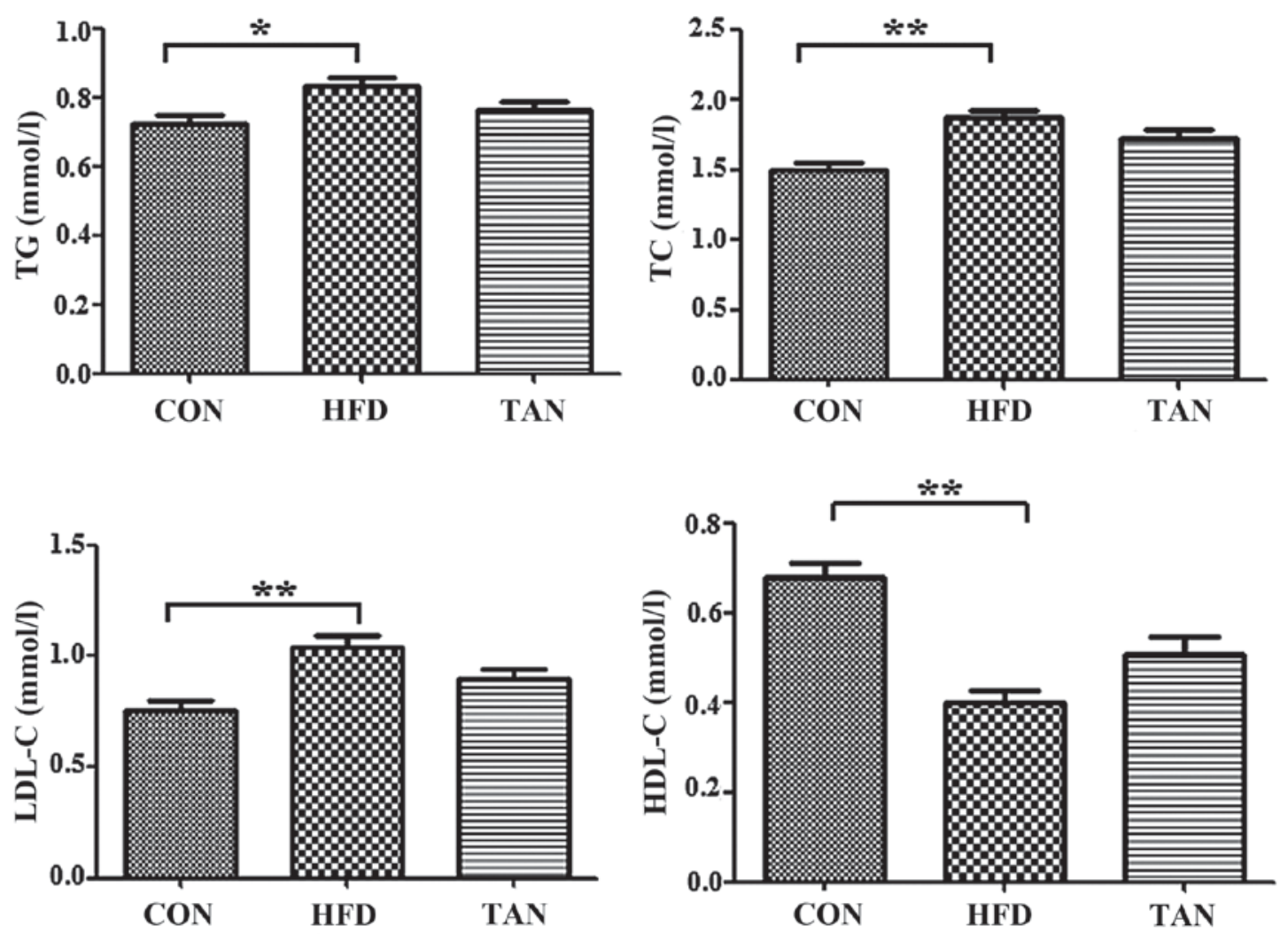

Figure 1. Serum lipid profile. TG, TC, HDL-C and LDL-C levels were determined using corresponding test kits in the CON, HFD and TAN groups. Data are presented as the mean + standard deviation $(\mathrm{n}=6) .{ }^{*} \mathrm{P}<0.05 ;{ }^{* *} \mathrm{P}<0.01$. CON, control group; HFD, high-fat diet group; TAN, tanshinone II A treatment group; TG, thyroglobulin; TC, total cholesterol; HDL-C, high density lipoprotein cholesterol; LDL-C, low density lipoprotein cholesterol.

with $50 \%$ ethanol, rinsed with tap water, stained with hematoxylin, rinsed with tap water and mounted with glycerin jelly. Sections were subsequently examined and images were captured using a light microscope linked to a digital CCD camera at magnification, $\mathrm{x} 200$.

Hoechst staining. Liver samples that had been fixed with $4 \%$ paraformaldehyde were cut into $5-\mu \mathrm{m}$ thick sections, deparaffinized and rehydrated. The tissue was permeabilized with Triton X-100 for 15 min. Nuclei were counterstained with Hoechst 33258, according to the manufacturer's instructions using an Apoptotic cell Hoechst 33258 Detection kit (Nanjing KeyGen Biotech Co., Ltd., Nanjing, China). Slides were examined for Hoechst using a fluorescence microscope at $350 \mathrm{~nm}$ and magnification, $\mathrm{x} 200$.

$R N A$ isolation and $R T-q P C R$. Total RNA was isolated from liver tissue using TRIzol reagent and reverse transcribed into cDNA using the miScript II RT kit (Qiagen GmbH, Hiden, Germany). Primers were chosen to detect the amplification of the chosen gene and were designed as follows: B cell lymphoma 2 associated protein $\mathrm{X}$ (Bax), forward 5'-GCAAACTGG TGCTCAAGG-3' and reverse 5'-CGTCCCGAAGTAGGA AAGG-3'; B cell lymphoma-2 (Bcl-2), forward 5'-CGGGAG AACAGGGTATGA-3' and reverse 5'-CAGGCTGGAAGG AGAAGAT-3'; caspase 3, forward 5'-CTGGACTGCGGT ATTGAG-3' and reverse 5'-GGGTGCGGTAGAGTAAGC-3'; and GAPDH, forward 5'-TGTGTCCGTCGTGGATCTGA-3' and reverse 5'-TTGCTGTTGAAGTCGCAGGAG-3'. The reaction mix consisted of $2 \mu \mathrm{l}$ cDNA, $0.5 \mu 1$ forward and reverse primer mix (10 $\mu \mathrm{M}$ of each), $12.5 \mu \mathrm{l} \mathrm{SYBR}$ Premix Ex Taq II (Takara Biotechnology Co., Ltd.) in a final volume of $25 \mu \mathrm{l}$. The following thermal conditions were used for all PCR reactions: $2 \mathrm{~min}$ at $95^{\circ} \mathrm{C}$, followed by 40 cycles of $30 \mathrm{sec}$ at $95^{\circ} \mathrm{C}$ and $30 \mathrm{sec}$ at $60^{\circ} \mathrm{C}$. RT-qPCR was performed in triplicate using SYBR Green Master Mix (Takara Biotechnology Co., Ltd.) on an ABI 7500 PCR Sequence Detector (Applied Biosystems; Thermo Fisher Scientific Inc., Waltham, MA, USA). Data were analyzed using the SDS software (version 2.0.6; Applied Biosystems; Thermo Fisher Scientific, Inc.). The fold-change in gene expression was determined by the $2^{\Delta \Delta \mathrm{Cq}}$ (23) method with GAPDH (housekeeping gene) as an internal control. The size of the amplified products were indicated to be 149, 149 and 102 bp for Bax, Bcl-2 and caspase 3, respectively.

Determination of hepatic ROS. Levels of ROS in liver slices were determined by the oxidation of dihydroethidium (DHE). Frozen sections of liver (5- $\mu \mathrm{m}$ thick) were incubated for $30 \mathrm{~min}$ at $37^{\circ} \mathrm{C}$ in $1 \mathrm{ml}$ of 4-(2-hydroxyethyl)-1-piperazineethanesulphonic acid buffer containing $50 \mu \mathrm{M}$ of DHE. Frozen sections were subsequently washed three times in phosphate-buffered saline. DHE was oxidized by ROS to produce fluorescent ethidium, which is able to bind to nucleic acids, thus further staining the nucleus a bright fluorescenct red. The formation of ethidium was monitored by fluorescence inversion microscopy (magnification, x200) with excitation and emission wavelengths of 480 and $590 \mathrm{~nm}$, respectively. 
A
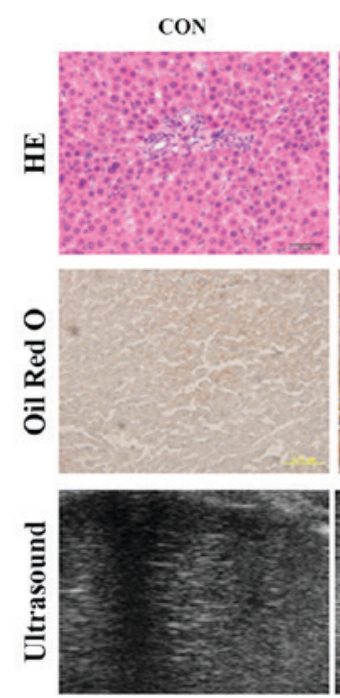

HFD
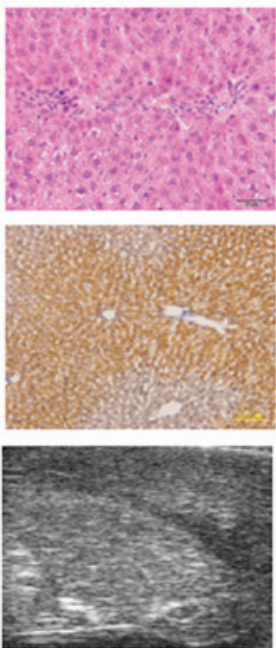

TAN
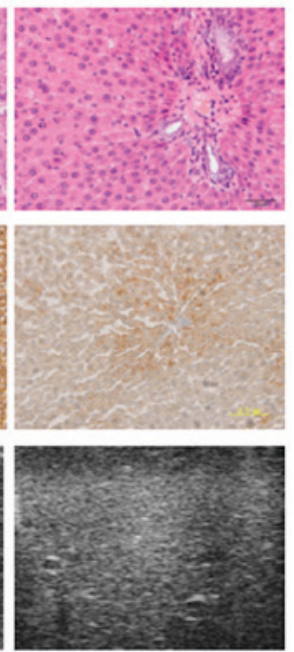

B

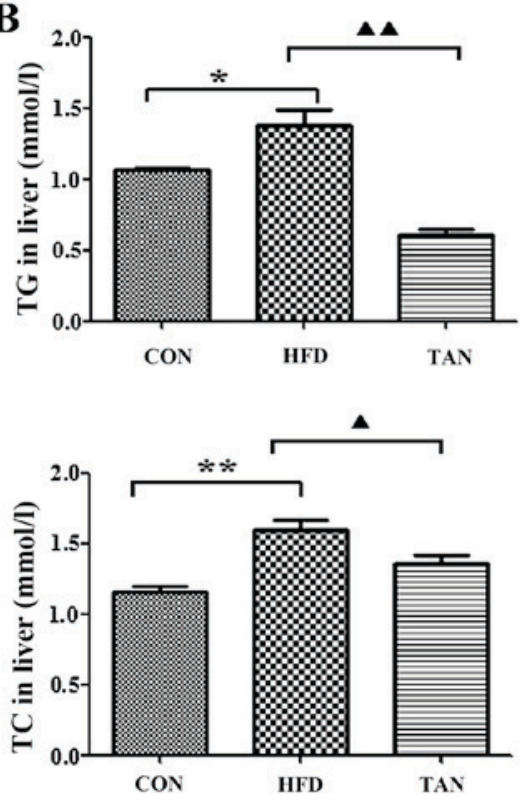

Figure 2. Differences in morphology and lipid deposition in rats from the CON, HFD and TAN groups. (A) Morphological observation of rat hepatocytes in CON, HFD and TAN groups by HE staining, Oil Red O staining and high-resolution ultrasound. (B) TG and TC were measured by the COD-CE-PAP and GPO-PAP methods, respectively, in the livers of CON, HFD and TAN rats. Data are presented as the mean + standard deviation $(\mathrm{n}=3)$. ${ }^{*} \mathrm{P}<0.05 ;{ }^{* *} \mathrm{P}<0.01$; ${ }^{\Delta} \mathrm{P}<0.05 ;{ }^{\boldsymbol{\Delta}} \mathrm{P}<0.01$. CON, control group; HFD, high-fat diet group; TAN, tanshinone II A treatment group; TG, thyroglobulin; TC, total cholesterol; HE, hematoxylin and eosin.
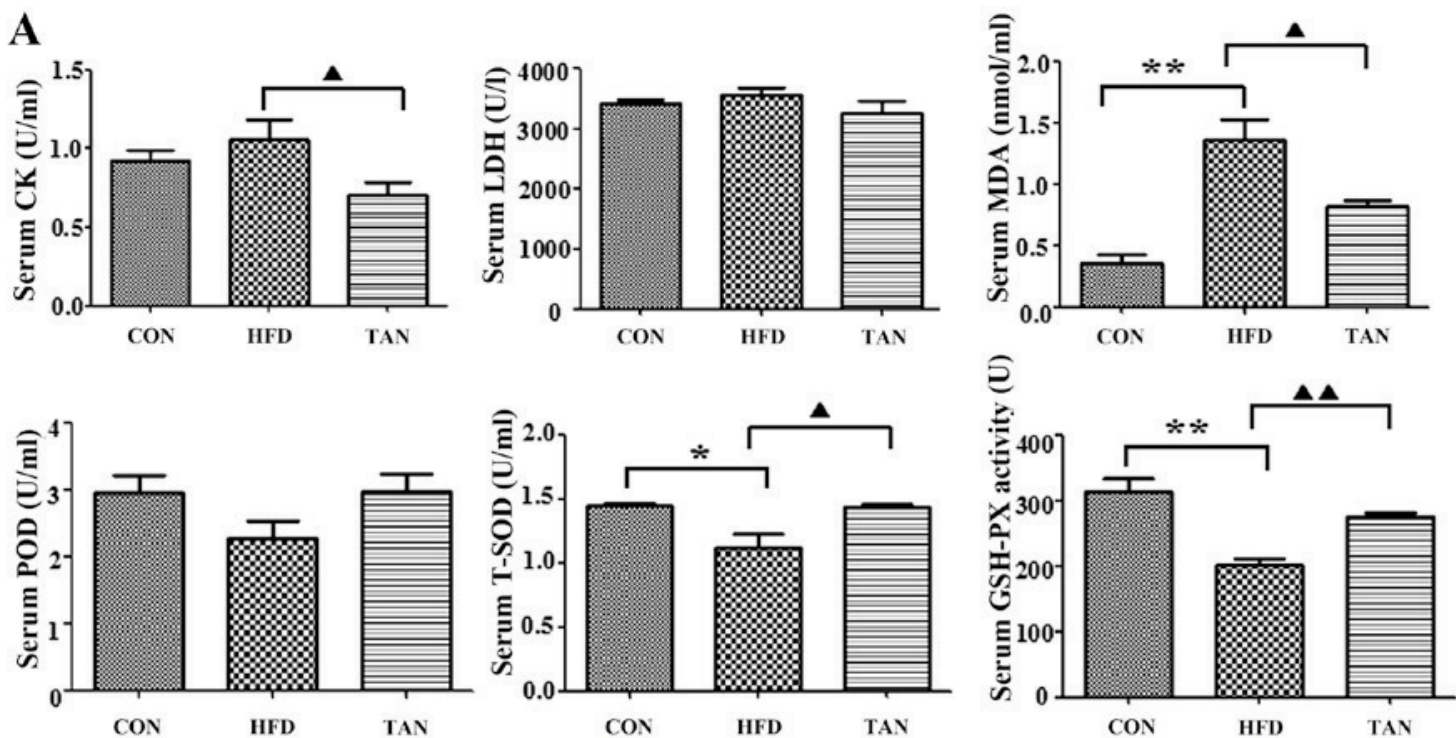

\section{B}
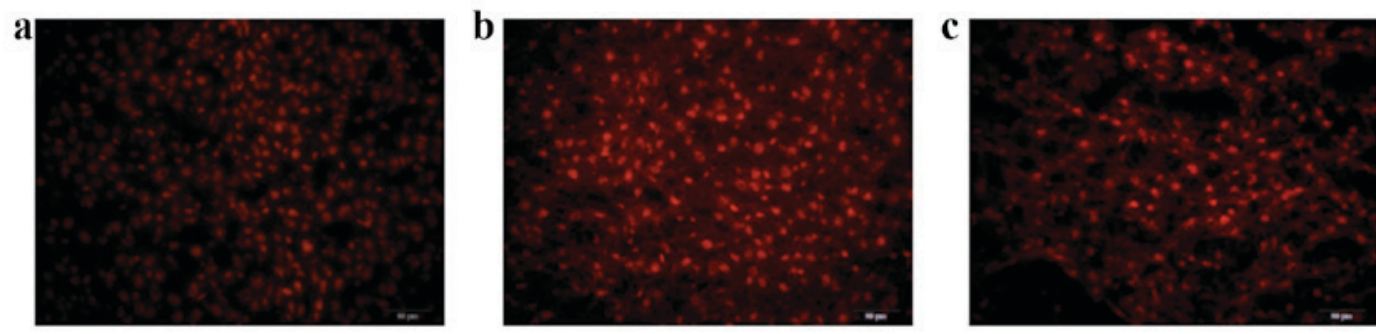

Figure 3. Effects of tanshinone IIA on high-fat diet-induced oxidative stress in the liver of rats. (A) Quantification of CK, LDH, MDA, POD, T-SOD and GSH-PX in the serum of rats from the CON, HFD and TAN groups. Rats were treated and the contents were subsequently assayed. Data are presented as the mean + standard deviation $(\mathrm{n}=3) .{ }^{*} \mathrm{P}<0.05 ;{ }^{* *} \mathrm{P}<0.01 ;{ }^{\mathbf{\Lambda}} \mathrm{P}<0.05 ;{ }^{\boldsymbol{\Delta}}{ }^{\boldsymbol{}} \mathrm{P}<0.01$. (B) Detection of ROS in the liver of rats from the (a) CON group; (b) HFD group; and (c) TAN group. Rats were treated and dihydroethidium fluorescence was detected. Red color fluorescence represents ROS. CK, creatine kinase; LDH, lactate dehydrogenase; MDA, malondialdehyde; POD, peroxidase, T-SOD, total superoxide dismutase; GSH-PX, glutathione peroxidase; CON, control group; HFD, high-fat diet group; TAN, tanshinone II A treatment group; ROS, reactive oxygen species. 
CON

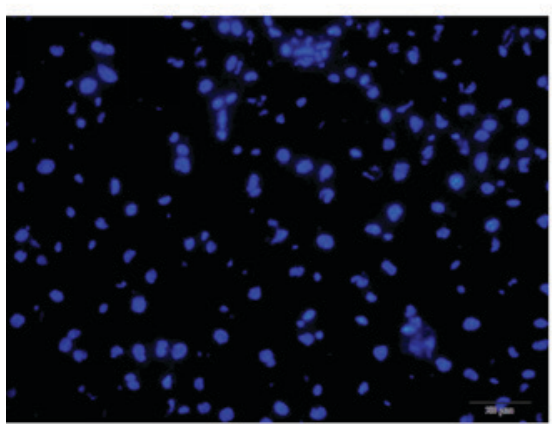

HLP

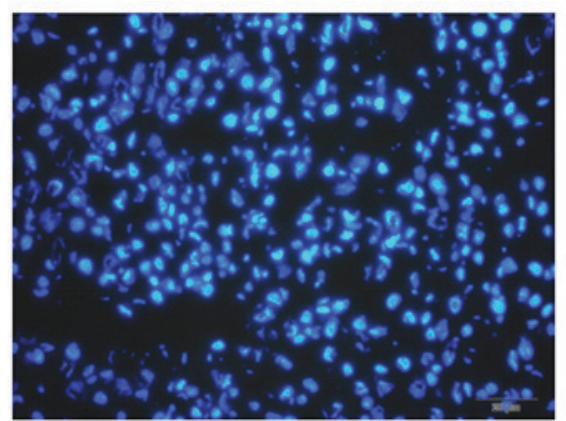

TAN

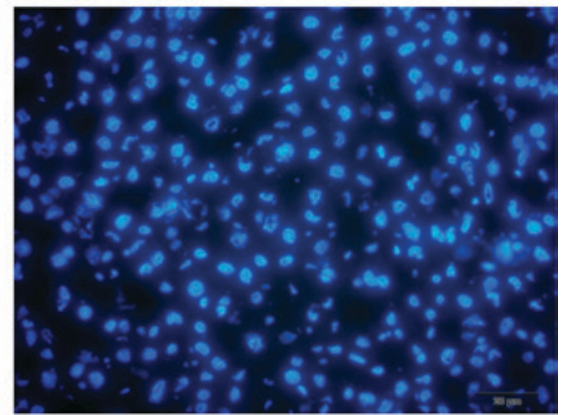

Figure 4. Hoechst assay in the liver of rats in the CON group ( $n=3)$, HFD group ( $n=3)$ and TAN group ( $n=3)$. Blue colour indicates apoptotic cells. CON, control group; HFD, high-fat diet group; TAN, tanshinone II A treatment group.

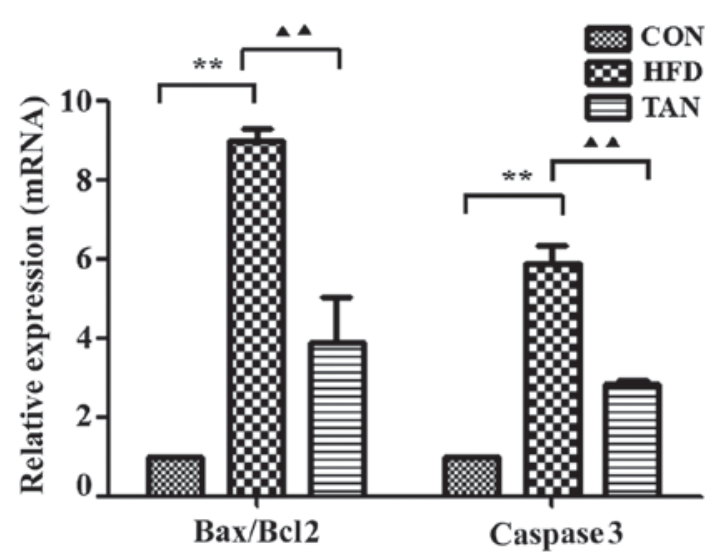

Figure 5. Apoptosis-related gene expression in the liver of rats. Expression levels of apoptosis-related genes, $\mathrm{Bax}, \mathrm{Bcl}-2$ and caspase 3 , in rat livers were analyzed by reverse transcription-quantitative polymerase chain reaction in CON, HFD and TAN groups. Data are presented as mean + standard deviation (n=3). ${ }^{* *} \mathrm{P}<0.01 ;{ }^{\mathbf{\Lambda}} \mathrm{P}<0.01$. CON, control group; HFD, high-fat diet group; TAN, tanshinone II A treatment group; Bcl-2, B cell lymphoma-2; $\mathrm{Bax}, \mathrm{Bcl}-2$ associated protein $\mathrm{X}$.

Protein extraction and western blotting. Total cellular protein was extracted from liver tissues using RIPA lysis buffer (Beyotime Institute of Biotechnology). Protein concentration was measured using a BCA protein assay kit (Beijing Dingguo Changsheng Biotechnology Co., Ltd., Beijing, China). To assess the protein expression levels of Bax, Bcl-2, and caspase 3 proteins, $80 \mu \mathrm{g}$ of protein samples were separated by $10 \%$ SDS-PAGE and transferred onto polyvinylidene difluoride membranes (Merck Millipore, Darmstadt, Germany). The membranes were incubated at $4^{\circ} \mathrm{C}$ overnight with primary antibodies against caspase 3 (1:800, 9662, Cell Signaling Technology, Inc., Danvers, MA, USA), Bax, Bcl-2 and GAPDH (1:500; sc-70407, sc-23960 and sc-32233; all Santa Cruz Biotechnology, Inc., Dallas, TX, USA) and subsequently with a secondary horseradish peroxidase-conjugated goat anti-rabbit and anti-mouse antibody (1:4,000, both Santa Cruz Biotechnology, Inc.) at room temperature for $1 \mathrm{~h}$. Protein bands were visualized using an enhanced chemiluminescence detection kit (Thermo Fisher Scientific, Inc.) and exposed to $\mathrm{X}$-ray film. Protein expression was normalized to the levels of GAPDH.
Statistical analysis. Data are presented as the mean + standard deviation. Differences among the groups were evaluated with the analysis of variance test for multiple groups, using GraphPad Prism 5.0 software (GraphPad Software, Inc., La Jolla, CA, USA). P $<0.05$ was considered to indicate a statistically significant difference.

\section{Results}

Serum lipid levels and lipid deposition in the liver. Compared with the CON group, the rats in the HFD group exhibited significantly increased levels of serum TG $(\mathrm{P}<0.05)$, TC $(\mathrm{P}<0.01)$ and LDL-C $(\mathrm{P}<0.01)$, and significantly decreased levels of serum HDL-C (P<0.01; Fig. 1). However, the TAN group did not indicate any significant difference in lipid profiles when compared with the HFD group (Fig. 1). Furthermore, hematoxylin and eosin-stained liver sections indicated severe hepatic steatosis in the HFD group (Fig. 2A). Compared with the CON group, the hepatocytes from the HFD group were swollen and round with a smaller cytoplasm and contained large lipid droplets. Furthermore, lipid vacuoles of different sizes and inflammatory cells were observed in hematoxylin and eosin-stained sections from the HFD group. In several hepatocytes, the nucleus appeared to be pushed to the side towards the cell membrane. The TAN group exhibited a reduction in hepatic steatosis, cell swelling and hepatocyte size in a number of lipid vacuoles when compared with HFD group (Fig. 2A). Oil Red O staining revealed that the hepatocytes in the HFD group contained a markedly increased number of lipid droplets when compared with the CON group. However, the number of lipid droplets was markedly decreased in the hepatocytes of the TAN group when compared with the HFD group. Compared with the homogeneous liver parenchyma of the HFD group, ultrasound examination of the liver of HFD rats indicated increased echogenicity of the liver parenchyma, characteristic of liver steatosis and heterogeneous parenchyma (24). The echo level of the liver parenchyma was revealed to be decreased in the TAN group when compared with the HFD group (Fig. 2A). Additionally, when compared with the CON group, the livers of rats in the HFD group exhibited significantly increased levels of TG $(\mathrm{P}<0.05)$ and TC $(\mathrm{P}<0.01)$; however, significantly decreased levels of TG 

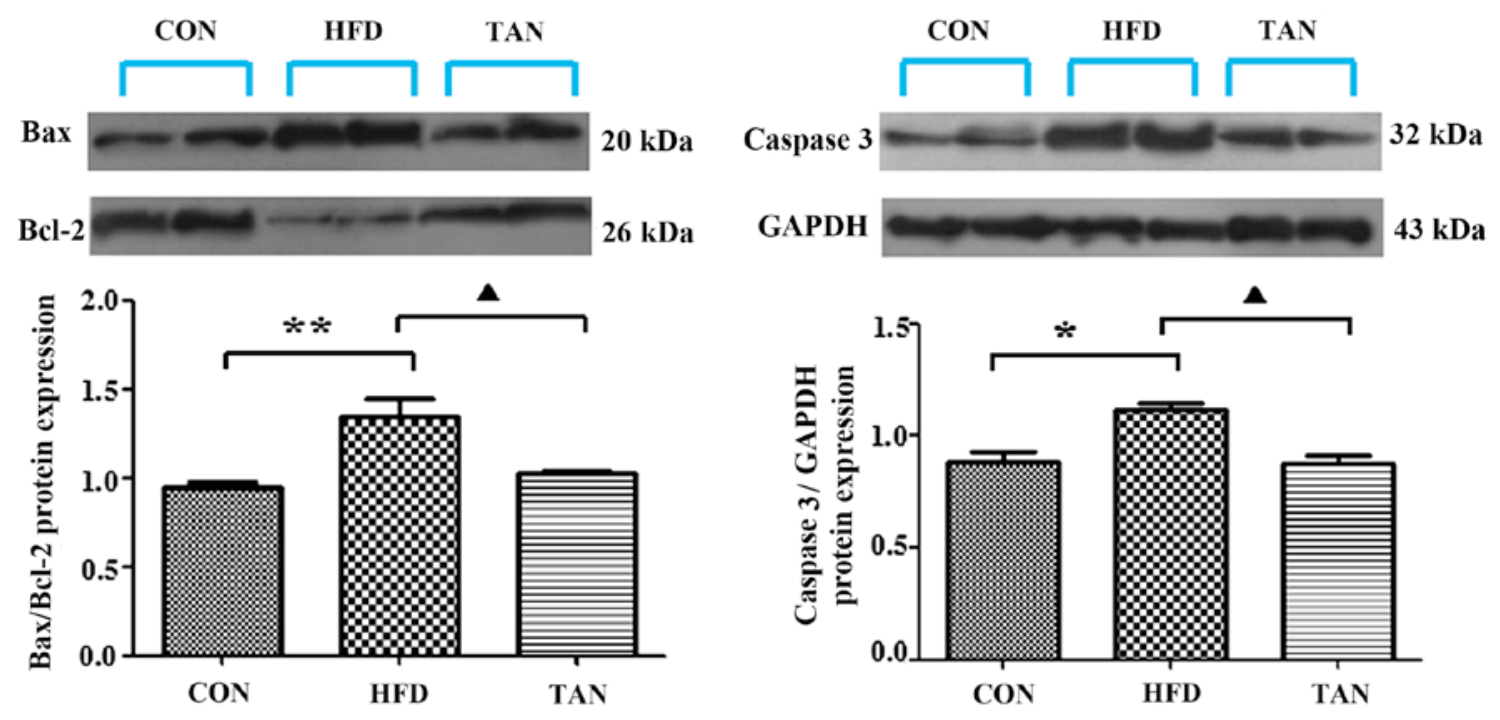

Figure 6. Apoptosis-related protein expression in the liver of rats. Expression levels of apoptosis-related proteins, Bax, Bcl-2 and caspase 3, in rat livers were analyzed by western blotting in the CON, HFD and TAN groups. Data are presented as the mean + standard deviation $(n=3)$. " $\mathrm{P}<0.05 ;{ }^{* * *} \mathrm{P}<0.01$; ${ }^{\mathbf{A}} \mathrm{P}<0.05$. CON, control group; HFD, high-fat diet group; TAN, tanshinone II A treatment group; Bcl-2, B cell lymphoma-2; Bax, Bcl-2 associated protein X.

$(\mathrm{P}<0.01)$ and TC $(\mathrm{P}<0.05)$ were indicated in the TAN group when compared with the HFD group (Fig. 2B).

Effect of TSIIA on antioxidant enzymes and the activity of CK and $L D H$. Compared with the CON group, MDA content in the HFD group was significantly elevated $(\mathrm{P}<0.01$; Fig. $3 \mathrm{~A})$, whereas the TAN group exhibited significantly decreased MDA content when compared with the HFD group $(\mathrm{P}<0.05)$. A significant decrease in T-SOD $(\mathrm{P}<0.05)$ and GSH-PX $(\mathrm{P}<0.01)$ was observed in the HFD group when compared with the CON group; however, significantly increased levels of T-SOD $(\mathrm{P}<0.05)$ and GSH-PX $(\mathrm{P}<0.01)$ were exhibited in the TAN group compared with the HFD group, indicating treatment with TSIIA reversed this effect. The level of POD activity was not significantly altered in all three groups. A comparison of the serum-marker enzymes LDH and CK, revealed a significant increase in $\mathrm{CK}$ activity $(\mathrm{P}<0.01)$ in the HFD group when compared with the TAN group; however, LDH activity was unchanged across all three groups.

Inhibitory effect of TSIIA on HFD-induced oxidative stress in the liver. Effects of TSIIA on HFD-induced oxidative stress in the liver of rats were indicated by fluorescence microscopy. Compared with the CON group, the HFD group exhibited markedly increased dihydroethidium fluorescence, which indicated increased ROS content in the liver. However, the level of fluorescence from livers of TAN group rats was indicated to be decreased when compared with the HFD group (Fig. 3B).

Effect of TIIA on liver cell apoptosis. To determine whether HFD induces apoptosis in liver cells and to explore whether apoptosis may be ameliorated by TSIIA treatment, rates of liver cell apoptosis were quantified via Hoechst assay analysis. The Hoechst assay revealed that the HFD group displayed a markedly increased rate of apoptosis when compared with the CON group. Furthermore, the TAN group exhibited a markedly decreased rate of apoptosis when compared with the HFD group, which indicated that TSIIA treatment was able to markedly reduce the rate of apoptosis, almost to the level observed in the CON group (Fig. 4).

Apoptosis-related gene and protein expression levels in the liver of rats. The ratio between the pro-apoptotic protein, Bax, and the anti-apoptotic protein, $\mathrm{Bcl}-2$, in addition to the expression levels of caspase 3, are important apoptotic indicators $(25,26)$. The results of RT-qPCR and western blotting showed that $\mathrm{Bax} / \mathrm{Bcl}-2 \mathrm{mRNA}$ and protein expression levels in the liver were significantly increased in the HFD group when compared with the CON group; however, these mRNA and protein expression levels were significantly decreased in the TAN group when compared with the HFD group $(\mathrm{P}<0.01$; Figs. 5 and 6). Furthermore, caspase $3 \mathrm{mRNA}$ and protein expression levels were significantly increased in the HFD group when compared with the CON group and these expression levels were significantly decreased in the TAN group when compared with the HFD group $(\mathrm{P}<0.05$; Figs. 5 and 6$)$.

\section{Discussion}

TSIIA is a major component of Salvia miltiorrhiza Bge (14). Previous studies have indicated that TSIIA possesses a range of biological properties $(15,16,27)$, predominantly based on its anti-oxidative effects (18). In the present study, the effect of TSIIA on liver steatosis was assessed in vivo. TSIIA was identified to reduce lipid deposition in the liver and ameliorate liver steatosis; however, this effect was not based on alterations of serum lipid levels, despite 3 months of treatment. This suggests that actions other than the lipid-lowering effect of TSIIA are responsible for the reduction in liver steatosis exhibited in the present study.

It has been previously reported that oxidative stress is associated with the pathogenesis of NAFLD $(28,29)$. Similarly, the present results indicated that the ROS levels, which are typically generated in a HFD (30), increased in rats in the HFD 
group. Correlating with increased ROS, the present findings demonstrated increased levels of lipid peroxide products, in the form of MDA, and decreased T-SOD and GSH-PX were exhibited in rats of the HFD group. As a result, the present findings indicated that the hepatocytes of fatty liver rats were damaged, through increased liver steatosis and apoptosis.

Apoptosis is an active process of cell suicide. Under physiological conditions, it can clear senescent and injured cells to maintain the normal liver volume. However, dysregulation of apoptosis may result in liver diseases $(31,32)$. Feldstein et al $(33,34)$ considered that hepatocyte apoptosis was the distinguishing feature of NAFLD and increased with the development of fatty liver. Furthermore, Murakawa et al (35) and other previous studies $(36,37)$ demonstrated that antioxidants had anti-apoptotic effects and implied that TSIIA may inhibit apoptosis due to its antioxidant activity. The present study identified that the rate of apoptosis was significantly increased in HFD group rats when compared to the CON group, by Hoechst staining. Moreover, the present findings further supported these effects at the molecular level by investigating Bax and Bcl-2 mRNA and protein expression. Bax and Bcl-2 are apoptosis-related genes; Bax is a pro-apoptotic factor, whereas Bcl-2 has anti-apoptotic activity (38). Xie et al (39) and McClintock et al (40) suggested that the survivability of cells post-apoptosis stimulation is dependent on the ratio of $\mathrm{Bax} / \mathrm{Bcl}-2$. Caspase 3, a cysteine protease, is able to promote apoptosis as a key protease (41). The present findings indicated an increase of the Bax/Bcl-2 ratio and caspase 3 mRNA and protein expression levels in the HFD group. However, these negative effects of a HFD on rat livers were reversed with TSIIA treatment, as indicated by the results from the TAN group. The present results indicate that enhanced anti-oxidative and anti-apoptotic capability may partially explain the protective effect of TSIIA on NAFLD.

Although TSIIA has no effect on serum lipid profiles, we found that attenuation of oxidative stress by TSIIA contributes to the amelioration of hepatic steatosis. In addition, the enhanced antioxidant capacity may account for the inhibition of apoptosis.

In summary, the present study showed that TSIIA improved the antioxidant capacity of the liver, leading to decreased apoptosis and improved hepatic steatosis in a rat model of fatty liver. In future studies, we plan to focus on the anti-apoptotic mechanisms of antioxidants, which may provide a potential target in the prevention and treatment of NAFLD.

\section{Acknowledgements}

The present work was supported by the National Program on Key Basic Research Project (973 Program) (grant no. 2013CB531704), the National Natural Science Foundation of China (grant no. 81774022) and the Key Laboratory of Ministry of Education for TCM Viscera-State Theory and Applications, Liaoning University of Traditional Chinese Medicine of China (grant no. 2014348).

\section{References}

1. Sinha N and Dabla PK: Oxidative stress and antioxidants in hypertension-A current review. Curr Hypertens Rev 11: 132-142, 2015.
2. Ibrahim W, Lee US, Yeh CC, Szabo J, Bruckner G and Chow CK: Oxidative stress and antioxidant status in mouse liver: Effects of dietary lipid, vitamin E and iron. J Nutr 127: 1401-1406, 1997.

3. Delbosc S, Paizanis E, Magous R, Araiz C, Dimo T, Cristol JP, Cros G and Azay J: Involvement of oxidative stress and NADPH oxidase activation in the development of cardio-vascular complications in a model of insulin resistance, the fructose-fed rat. Atherosclerosis 179: 43-49, 2005.

4. Shin MH, Moon YJ, Seo JE, Lee Y, Kim KH and Chung JH: Reactive oxygen species produced by NADPH oxidase, xanthine oxidase, and mitochondrial electron transport system mediate heat shock-induced MMP-1 and MMP-9 expression. Free Radic Biol Med 44: 635-645, 2008.

5. Zhao ZQ: Oxidative stress-elicited myocardial apoptosis during reperfusion. Curr Opin Pharmacol 4: 159-165, 2004.

6. Wang K: Molecular mechanisms of hepatic apoptosis. Cell Death Dis 5: e996, 2014.

7. Sies H: What is oxidative stress? Oxidative Stress Vascular Dis 224: 1-8, 2000.

8. Merker K, Stolzing A and Grune T: Proteolysis, caloric restriction and aging. Mech Ageing Dev 122: 595-615, 2001.

9. Smith BW and Adams LA: Non-alcoholic fatty liver disease. Crit Rev Clin Lab Sci 48: 97-113, 2011.

10. Castelli WP, Garrison RJ, Wilson PW, Abbott RD, Kalousdian S and Kannel WB: Incidence of coronary heart disease and lipoprotein cholesterol levels. The Framingham study. JAMA 20: 2835-2838, 1986.

11. Vendemiale G, Grattagliano I and Altomare E: An update on the role of free radicals and antioxidant defense in human disease. Int J Clin Lab Res 29: 49-55, 1999.

12. Aboelwafa HR and Yousef HN: The ameliorative effect of thymol against hydrocortisone-induced hepatic oxidative stress injury in adult male rats. Biochem Cell Biol 93: 282-289, 2015.

13. Duan L, Xiong XJ and Wang J: Activating blood circulation to remove stasis and therapeutic angiogenesis of coronary heart disease. Zhongguo Zhong Xi Yi Jie He Za Zhi 33: 1561-1566, 2013 (In Chinese).

14. Chen FY, Guo R and Zhang BK: Advances in cardiovascular effects of tanshinone II(A). Zhongguo Zhong Yao Za Zhi 40: 1649-1653, 2015 (In Chinese).

15. Gao S,Liu Z,LiH,LittlePJ,Liu P and Xu S: Cardiovascular actions and therapeutic potential of tanshinone IIA. Atherosclerosis 220: 3-10, 2012.

16. Shang $\mathrm{Q}, \mathrm{Xu} \mathrm{H}$ and Huang L: Tanshinone IIA: A promising natural cardioprotective agent. Evid Based Complement Alternat Med 2012: 716459, 2012.

17. Fu J, Huang H, Liu J, Pi R, Chen J and Liu P: Tanshinone IIA protects cardiac myocytes against oxidative stress-triggered damage and apoptosis. Eur J Pharmacol 30: 213-221, 2007.

18. Niu XL, Ichimori K, Yang X, Hirota Y, Hoshiai K, Li M and Nakazawa H: Tanshinone II-A inhibits low density lipoprotein oxidation in vitro. Free Radic Res 33: 305-312, 2000.

19. Tang FT, Cao Y, Wang TQ, Wang LJ, Guo J, Zhou XS, Xu SW, Liu WH, Liu PQ and Huang HQ: Tanshinone IIA attenuates atherosclerosis in ApoE(-/-) mice through down-regulation of scavenger receptor expression. Eur J Pharmacol 650: 275-284, 2011.

20. Jia LQ, Feng JY, Yang GL, Chen WN and Chen Y: Effect of tanshinone IIA on TLR4 and TNF- $\alpha$ of endothelial cells induced by LPS. Xi Bao Yu Fen Zi Mian Yi Xue Za Zhi 27: 733-735, 2011 (In Chinese).

21. Jia LQ, Yang GL, Ren L, Chen WN, Feng JY, Cao Y, Zhang L, Li XT and Lei P: Tanshinone IIA reduces apoptosis induced by hydrogen peroxide in the human endothelium-derived EA.hy 926 cells. J Ethnopharmacol 143: 100-108, 2012.

22. Jia LQ, Zhang N, Xu Y, Chen WN, Zhu ML, Song N, Ren L, Cao HM, Wang JY and Yang GL: Tanshinone IIA affects the HDL subfractions distribution not serum lipid levels: Involving in intake and efflux of cholesterol. Arch Biochem Biophys 592: 50-59, 2016.

23. Livak KJ and Schmittgen TD: Analysis of relative gene expression data using real-time quantitative PCR and the 2(-Delta Delta C(T)) method. Methods 25: 402-408, 2001.

24. Pereira ENGDS; Silvares RR, Flores EEI, Rodrigues KL, Ramos IP, da Silva IJ, Machado MP, Miranda RA, Pazos-Moura CC, Gonçalves-de-Albuquerque CF, et al: Hepatic microvascular dysfunction and increased advanced glycation end products are components of non-alcoholic fatty liver disease. PLoS One 12: e0179654, 2017. 
25. Jarskog LF, Selinger ES, Lieberman JA and Gilmore JH Apoptotic proteins in the temporal cortex in schizophrenia: High $\mathrm{Bax} / \mathrm{Bcl}-2$ ratio without caspase-3 activation. Am J Psychiatry 161: 109-115, 2004.

26. Peiró G, Diebold J, Baretton GB, Kimmig R and Löhrs U: Cellular apoptosis susceptibility gene expression in endometrial carcinoma: Correlation with Bcl-2, Bax, and caspase-3 expression and outcome. Int J Gynecol Pathol 20: 359-367, 2001.

27. Kimm HH, Kim JH, Kwak HB, Huang H, Han SH, Ha H, Lee SW, Woo ER and Lee ZH: Inhibition of osteoclast differentiation and bone resorption by tanshinone IIA isolated from Salvia miltiorrhiza Bunge. Biochem Pharmacol 67: 1647-1656, 2004.

28. Day CP and James OF: Steatohepatitis: A tale of two 'hits'? Gastroenterology 114: 842-845, 1998.

29. Wu D, Zheng N, Qi K, Cheng H, Sun Z, Gao B, Zhang Y, Pang W, Huangfu C, Ji S, et al: Exogenous hydrogen sulfide mitigates the fatty liver in obese mice through improving lipid metabolism and antioxidant potential. Med Gas Res 5: 1,2015.

30. Das N, Ganguli D and Dey S: Moringa oleifera Lam. seed extract prevents fat diet induced oxidative stress in mice and protects liver cell-nuclei from hydroxyl radical mediated damage. Indian J Exp Biol 53: 794-802, 2015.

31. Rust C and Gores GJ: Apoptosis and liver disease. Am J Med 108: $567-574,2000$

32. Benedetti A and Marucci L: The significance of apoptosis in the liver. Liver 19: 453-463, 1999.

33. Feldstein AE, Canbay A, Angulo P, Taniai M, Burgart LJ, Lindor KD and Gores GJ: Hepatocyte apoptosis and fas expression are prominent features of human nonalcoholic steatohepatitis. Gastroenterology 125: 437-443, 2003.

34. Feldstein AE, Canbay A, Guicciardi ME, Higuchi H, Bronk SF and Gores GJ: Diet associated hepatic steatosis sensitizes to Fas mediated liver injury in mice. J Hepatol 39: 978-983, 2003.
35. Murakawa M, Jung SK, Lijima $K$ and Yonehara S: Apoptosis-inducing protein, AIP, from parasite-infected fish induces apoptosis in mammalian cells by two different molecular mechanisms. Cell Death Differ 8: 298-307, 2001.

36. Emanuele S, Galvaruso G, Lauricella M, Giuliano M, Bellavia G, D'Anneo A, Vento R and Tesoriere G: Apoptosis induced in hepatoblastoma HepG2 cells by the proteasome inhibitor MG132 is associated with hydrogen peroxide production, expression of Bcl-XS and activation of caspase-3. Int J Oncol 21: 857-865, 2002.

37. Woo SH, Park IC, Park MJ, Lee HC, Lee SJ, Chun YJ, Lee SH, Hong SI and Rhee CH: Arsenic trioxide induces apoptosis through a reactive oxygen species-dependent path way and loss of mitochondrial membrane potential in HeLa cells. Int J Oncol 21: 57-63, 2002.

38. Walensky LD: BCL-2 in the crosshairs: Tipping the balance of life and death. Cell Death Differ 13: 1339-1350, 2006.

39. Xie Z, Koyama T, Suzuki J, Fujii Y, Togashi H, Sawa H and Nagashima K: Coronary reperfusion following ischemia: Different expression of bcl-1 and bax proteins, and cardiomyocyte apoptosis. Jpn Heart J 42: 759-770, 2001.

40. McClintock DS, Santore MT, Lee VY, Brunelle J, Budinger GR, Zong WX, Thompson CB, Hay N and Chandel NS: Bcl-2 family members and functional electron transport chain regulate oxygen deprivation-induced cell death. Mol Cell Biol 22: 94-104, 2002.

41. Nakagawa Y: Initiation of apoptotic signal by the peroxidation of cardiolipin of mitochondria. Ann N Y Acad Sci 1011: 177-184, 2004. 